\title{
Chain Graph Interpretations and their Relations
}

\author{
Dag Sonntag and Jose M. Peña \\ ADIT, IDA, Linköping University, Sweden \\ dag.sonntag@liu.se, jose.m.pena@liu.se
}

\begin{abstract}
This paper deals with different chain graph interpretations and the relations between them in terms of representable independence models. Specifically, we study the Lauritzen-Wermuth-Frydenberg, Andersson-Madigan-Pearlman and multivariate regression interpretations and present the necessary and sufficient conditions for when a chain graph of one interpretation can be perfectly translated into a chain graph of another interpretation. Moreover, we also present a feasible split for the Andersson-Madigan-Pearlman interpretation with similar features as the feasible splits presented for the other two interpretations.

Keywords: Chain Graphs, Lauritzen-Wermuth-Frydenberg interpretation, Andersson-Madigan-Pearlman interpretation, multivariate regression interpretation.
\end{abstract}

\section{Introduction}

Today there exist mainly three interpretations of chain graphs (CGs). These are the Lauritzen-Wermuth-Frydenberg (LWF) interpretation presented by Lauritzen, Wermuth and Frydenberg in the late eighties [6, 7], the Andersson-MadiganPearlman (AMP) interpretation presented by Anderson, Madigan and Pearlman in 2001 [2] and the multivariate regression (MVR) interpretation presented by Cox and Wermuth in the nineties [3,4]. A fourth interpretation of CGs can also be found in a study by Drton [5] but this interpretation has not been further studied and will not be discussed in this paper.

Each interpretation has a different separation criterion and do therefore represent different independence models. So far most papers have studied the different interpretations independently with a few exceptions such as the study of discrete CG models by Drton [5] and the study of CGs representing Gaussian distributions by Wermuth et al. [12]. Therefore it has not really been studied what differences and similarities that exist between the different interpretations in terms of representable independence models. Andersson et al. made a small study of this when they presented their new (AMP) interpretation and managed to show when the independence model of a CG of the AMP interpretation could be represented perfectly by a CG of the LWF interpretation. They did however not show when the opposite held and did no comparison with CGs of the MVR interpretation. Wermuth and Sadeghi did on the other hand present conditions for when a CG of the MVR interpretation could be translated into a CG of the LWF or AMP interpretation when they introduced regression graphs [11]. The 
conditions were however only necessary and sufficient if the two CGs contained the same connectivity components and not the more general case where the CGs could take any form.

In this paper we hope to fill this gap and hence the main contribution of this paper is a table where we show the necessary and sufficient conditions for when a CG of one interpretation can be perfectly translated into a CG of another interpretation. First we do however define a feasible split for the AMP interpretation, with similar features as the feasible splits shown for the LWF [10] and MVR [9] interpretation, that are used in these conditions. Hence this is our second contribution. Finally we also show that there for all three CG interpretations exists a minimal set of non-directed edges for each Markov equivalence class and that the CG containing these, and only these, non-directed edges can be reached through repeated feasible splits from any member of the class.

The remainder of the article is organized as follows. In the next section we present the notation we will use throughout the article. This is followed by the definitions of the feasible splits for each interpretation as well as the proof that the feasible split for CGs of the AMP interpretation is sound. In section 4 we start by presenting the conditions of when a CG of one interpretation can be perfectly represented by a CG of another interpretation. This is then followed by the proofs that these conditions are sound.

\section{Notation}

All graphs are defined over a finite set of variables $V$. If a graph $G$ contains an edge between two nodes $V_{1}$ and $V_{2}$, we denote with $V_{1} \rightarrow V_{2}$ a directed edge, with $V_{1} \leftrightarrow V_{2}$ a bidirected edge and with $V_{1}-V_{2}$ an undirected edge. By $V_{1} \leftrightarrow V_{2}$ we mean that either $V_{1} \rightarrow V_{2}$ or $V_{1} \leftrightarrow V_{2}$ is in $G$. By $V_{1} \multimap V_{2}$ we mean that either $V_{1} \rightarrow V_{2}$ or $V_{1}-V_{2}$ is in $G$. By $V_{1} \circ V_{2}$ we mean that there exists an edge between $V_{1}$ and $V_{2}$ in $G$ while we with $V_{1} \cdots V_{2}$ mean that there might or might not exist an edge between $V_{1}$ and $V_{2}$. By a non-directed edge we mean either a bidirected edge or an undirected edge. A set of nodes is said to be complete if there exist edges between all pairs of nodes in the set.

The parents of a set of nodes $X$ of $G$ is the set $p a_{G}(X)=\left\{V_{1} \mid V_{1} \rightarrow V_{2}\right.$ is in $G, V_{1} \notin X$ and $\left.V_{2} \in X\right\}$. The children of $X$ is the set $c h_{G}(X)=\left\{V_{1} \mid V_{2} \rightarrow V_{1}\right.$ is in $G, V_{1} \notin X$ and $\left.V_{2} \in X\right\}$. The spouses of $X$ is the set $\operatorname{sp}_{G}(X)=\left\{V_{1} \mid V_{1} \leftrightarrow V_{2}\right.$ is in $G, V_{1} \notin X$ and $\left.V_{2} \in X\right\}$. The neighbours of $X$ is the set $n b_{G}(X)=\left\{V_{1} \mid V_{1}-V_{2}\right.$ is in $G, V_{1} \notin X$ and $\left.V_{2} \in X\right\}$. The boundary of $X$ is the set $b d_{G}(X)=p a_{G}(X) \cup$ $n b_{G}(X) \cup s p_{G}(X)$. The adjacents of $X$ is the set $a d_{G}(X)=\left\{V_{1} \mid V_{1} \rightarrow V_{2}, V_{1} \leftarrow V_{2}\right.$, $V_{1} \leftrightarrow V_{2}$ or $V_{1}-V_{2}$ is in $G, V_{1} \notin X$ and $\left.V_{2} \in X\right\}$.

A route from a node $V_{1}$ to a node $V_{n}$ in $G$ is a sequence of nodes $V_{1}, \ldots, V_{n}$ such that $V_{i} \in \operatorname{ad}_{G}\left(V_{i+1}\right)$ for all $1 \leq i<n$. A path is a route containing only distinct nodes. The length of a path is the number of edges in the path. A path is called a cycle if $V_{n}=V_{1}$. A path is descending if $V_{i} \in p a_{G}\left(V_{i+1}\right) \cup s p_{G}\left(V_{i+1}\right) \cup n b_{G}\left(V_{i+1}\right)$ for all $1 \leq i<n$. A path $\pi=V_{1}, \ldots, V_{n}$ is minimal if there exists no other path $\pi_{2}$ between $V_{1}$ and $V_{n}$ st $\pi_{2} \subset \pi$ holds. The descendants of a set of nodes $X$ of $G$ is 
the set $\operatorname{de}_{G}(X)=\left\{V_{n} \mid\right.$ there is a descending path from $V_{1}$ to $V_{n}$ in $G, V_{1} \in X$ and $\left.V_{n} \notin X\right\}$. A path is strictly descending if $V_{i} \in p a_{G}\left(V_{i+1}\right)$ for all $1 \leq i<n$. The strict descendants of a set of nodes $X$ of $G$ is the set $\operatorname{sde}_{G}(X)=\left\{V_{n} \mid\right.$ there is a strict descending path from $V_{1}$ to $V_{n}$ in $G, V_{1} \in X$ and $\left.V_{n} \notin X\right\}$. The ancestors (resp. strict ancestors) of $X$ is the set $a n_{G}(X)=\left\{V_{1} \mid V_{n} \in d e_{G}\left(V_{1}\right), V_{1} \notin X, V_{n} \in X\right\}$ (resp. $\left.\operatorname{san}_{G}(X)=\left\{V_{1} \mid V_{n} \in \operatorname{sde} e_{G}\left(V_{1}\right), V_{1} \notin X, V_{n} \in X\right\}\right)$. A cycle is called a semidirected cycle if it is descending and $V_{i} \rightarrow V_{i+1}$ is in $G$ for some $1 \leq i<n$. A CG under the Lauritzen-Wermuth-Frydenberg (LWF) interpretation, denoted LWF $\mathrm{CG}$, contains only directed and undirected edges but no semi-directed cycles. Likewise a CG under the Andersson-Madigan-Perlman (AMP) interpretation, denoted AMP CG, is a graph containing only directed and undirected edges but no semi-directed cycles. A CG under the multivariate regression (MVR) interpretation, denoted MVR CG, is a graph containing only directed and bidirected edges but no semi-directed cycles. A connectivity component $C$ of a LWF CG or an AMP CG (resp. MVR CG) is a maximal (wrt set inclusion) set of nodes such that there exists a path between every pair of nodes in $C$ containing only undirected edges (resp. bidirected edges). We denote the set of all connectivity components in a CG $G$ by $c c(G)$ and the component to which a set of nodes $X$ belong in $G$ by $\operatorname{co}_{G}(X)$. A subgraph of $G$ is a subset of nodes and edges in $G$. A subgraph of $G$ induced by a set of its nodes $X$ is the graph over $X$ that has all and only the edges in $G$ whose both ends are in $X$. A bidirected flag is an induced subgraph of the form $X \leftrightarrow Y \leftrightarrow Z$ in a MVR CG. With the moral closure graph of a component $C$ in a LWF CG $G$, denoted $\left(G_{c l(C)}\right)^{m}$, we mean the subgraph of $G$ induced by $C \cup p a_{G}(C)$ where every edge have been made undirected and every pair of nodes in $p a_{G}(C)$ have been made adjacent with undirected edges.

Let $X, Y$ and $Z$ denote three disjoint subsets of $V$. We say that $X$ separated from $Y$ given $Z$ denoted as $X \perp_{G} Y \mid Z$ if the following criteria is met: If $G$ is a LWF CG then $X$ and $Y$ are separated given $Z$ iff there exists no route between $X$ and $Y$ such that every node in a non-collider section on the route is not in $Z$ and some node in every collider section on the route is in $Z$. A section of a route is a maximal (wrt set inclusion) non-empty set of nodes $B_{1} \ldots B_{n}$ such that the route contains the subpath $B_{1}-B_{2}-\ldots-B_{n}$. It is called a collider section if $B_{1} \ldots B_{n}$ together with the two neighbouring nodes in the route, $A$ and $C$, form the subpath $A \rightarrow B_{1}-B_{2}-\ldots-B_{n} \leftarrow C$. For any other configuration the section is a non-collider section. If $G$ is an AMP CG then $X$ and $Y$ is separated given $Z$ iff there exists no S-open path between $X$ and $Y$. A path is said to be $S$-open iff every non-head-no-tail node on the path is not in $Z$ and every head-no-tail node on the path is in $Z$ or $\operatorname{san}_{G}(Z)$. A node $B$ is said to be a head-no-tail in an AMP CG $G$ between two nodes $A$ and $C$ on a path if one of the following configurations exists in $G$ : $A \rightarrow B \leftarrow C, A \rightarrow B-C$ or $A-B \leftarrow C$. Moreover $G$ is also said to contain a triplex $(\{A, C\}, B)$ iff one such configuration exists in $G$ and $A$ and $C$ are not adjacent in $G$. For any other configuration the node $B$ is a non-collider. If $G$ is a MVR CG then $X$ and $Y$ are separated given $Z$ iff there exists no d-connecting path between $X$ and $Y$. A path is said to be $d$-connecting iff every non-collider on the path is not in $Z$ and every collider on the path is 
in $Z$ or $\operatorname{san}_{G}(Z)$. A node $B$ is said to be a collider in a MVR CG $G$ between two nodes $A$ and $C$ on a path if one of the following configurations exists in $G: A \rightarrow B \leftarrow C, A \rightarrow B \leftrightarrow C, A \leftrightarrow B \leftarrow C$ or $A \leftrightarrow B \leftrightarrow C$. For any other configuration the node $B$ is a non-collider.

The independence model $M$ induced by a graph $G$, denoted as $I(G)$ or $I_{P G M \text {-class }}(G)$, is the set of separation statements $X \perp_{G} Y \mid Z$ that hold in $G$ according to the interpretation to which $G$ belongs or the subscripted PGM-class. We say that two graphs $G$ and $H$ are Markov equivalent (under the same interpretation) or that they are in the same Markov equivalence class iff $I(G)=I(H)$.

\section{$3 \quad$ Feasible Splits}

For the LWF and MVR interpretation, operations for altering a CG structure without changing its Markov equivalence class have been presented [9, 10]. One such operation is called feasible split and is in this article used to prove certain theorems. Hence we repeat the definitions here. Moreover, we also present the corresponding operation, called feasible split for AMP CGs, for the AMP CG interpretation and prove that it is sound. Note that this is not the inverse operation to a legal merging presented in the deflagging procedure for AMP CGs by Roverto and Studený [8]. Their operation was applied to so called strong equivalence classes, not the more general Markov equivalence classes used here.

Definition 1. Feasible split for LWF CGs [10] A connectivity component $C$ of $C G G$ under the $L W F$ interpretation can be feasibly split into two disjoint sets $U$ and $L$ st $U \cup L=C$ by replacing every undirected edge between $U$ and $L$ with a directed edge orientated towards $L$ iff:

1. $\forall A \in n e_{G}(L) \cap U, p a_{G}(L) \subseteq p a_{G}(A)$

2. $n e_{G}(L) \cap U$ is complete

Definition 2. Feasible split for AMP CGs

$A$ connectivity component $C$ of $C G G$ under the $A M P$ interpretation can be feasibly split into two disjoint sets $U$ and $L$ st $U \cup L=C$ by replacing every undirected edge between $U$ and $L$ with a directed edge orientated towards $L$ iff:

1. $\forall A \in n e_{G}(L) \cap U, L \subseteq n e_{G}(A)$

2. $n e_{G}(L) \cap U$ is complete

3. $\forall B \in L, p a_{G}\left(n e_{G}(L) \cap U\right) \subseteq p a_{G}(B)$

Definition 3. Feasible split for MVR CGs [9]

A connectivity component $C$ of $C G G$ under the MVR interpretation can be feasible split into two disjoint sets $U$ and $L$ st $U \cup L=C$ by replacing every bidirected edge between $U$ and $L$ with a directed edge orientated towards $L$ iff:

1. $\forall A \in s p_{G}(U) \cap L, U \subseteq s p_{G}(A)$ holds

2. $\forall A \in s p_{G}(U) \cap L, p a_{G}(U) \subseteq p a_{G}(A)$ holds

3. $\forall B \in \operatorname{sp}_{G}(L) \cap U, s p_{G}(B) \cap L$ is a complete set 
Definition 4. Maximally orientated $C G$

A CG G (under any interpretation) is maximally orientated iff no feasible splits can be performed on $G$.

Lemma 1. A CG G of the AMP interpretation is in the same Markov equivalence class before and after a feasible split.

Proof. Assume the contrary. Let $G$ be a CG under the AMP interpretations and $G^{\prime}$ a graph st $G^{\prime}$ is $G$ with a feasible split performed upon it. $G$ and $G^{\prime}$ are in different Markov equivalence classes or $G^{\prime}$ is not a CG under the AMP interpretation iff (1) $G$ and $G^{\prime}$ does not have the same adjacencies, (2) $G$ and $G^{\prime}$ does not have the same triplexes or (3) $G^{\prime}$ contains semi-directed cycles.

First it is clear that $G$ and $G^{\prime}$ contains the same adjacencies since a feasible split does not change the adjacencies of any node in $G$. Secondly let us assume $G$ and $G^{\prime}$ does not have the same triplexes. First let us assume that $G^{\prime}$ contains a triplex $(\{X, Y\}, Z$ that does not exist in $G$. It is clear that such a triplex can only occur if $Z \in L$ since the only difference between $G$ and $G^{\prime}$ is that $G^{\prime}$ contains some directed edges orientated towards $L$ where $G$ contains undirected edges. It is clear that if the triplex is a flag then the one of the node $X$ or $Y$, let's say $X$, must be in $U$ and the other one, let's say $Y$, must be in $L$. However, according to condition $1 Y$ must be adjacent to $X$ which causes a contradiction. If the triplex is not a flag both $X$ and $Y$ must be in $U$. They also have to be in $n e_{G}(L)$, which, together with condition 2 , contradicts that they are not adjacent. Hence we have a contradiction for that $G^{\prime}$ contains a triplex that does not exist in $G$.

Secondly assume $G$ contains a triplex $(\{X, Y\}, Z)$ that does not exist in $G^{\prime}$. It is clear that this new triplex cannot be over a node in $L$ since these nodes only have edges orientated towards them. Instead assume $Z \in U$. This gives that one of the nodes $X$ or $Y$, let's say $X$, must be a parent of $Z$ and the other, let's say $Y$, must be in $L$. This does however contradict condition 3 , since every parent of $Z$ also must be a parent of $Y$, and hence $X$ and $Y$ must be adjacent. This gives us a contradiction.

Finally assume $G^{\prime}$ contain a semi-directed cycle. This means there exists two nodes $X$ and $Y$ st $X \in p a_{G^{\prime}}(Y)$ but $X \in d e_{G^{\prime}}(Y) \cup c o_{G^{\prime}}(Y)$. It is clear that $\forall A \in V \operatorname{de}_{G^{\prime}}(A) \subseteq d e_{G}(A)$ and $c_{G^{\prime}}(A) \subseteq \operatorname{co}_{G}(A)$ hold. Hence we must have that $X \in \operatorname{de}_{G}(Y) \cup c o_{G}(Y)$ also hold which, together with $\forall B \in V \backslash L$ $p a_{G^{\prime}}(B)=p a_{G}(B)$, means that $Y$ is in $L$ and since $\forall D \in L p a_{G^{\prime}}(D)=p a_{G}(D) \cup U$ holds $X$ must be in $U$. However, at the same time $\operatorname{co}_{G^{\prime}}(Y)=\operatorname{co}_{G} Y \backslash U$ and $d e_{G^{\prime}}(Y) \subseteq d e_{G} Y$ must hold and hence we have a contradiction.

A maximally orientated CG can be obtained from any member of its Markov equivalence class by performing feasible splits until no more feasible splits can be performed.

Theorem 1. A CG (under any interpretation) has the minimal set of nondirected edges for its Markov equivalence class if no feasible split is possible.

The following theorem shows that there may exist several maximally orientated CGs in a given Markov equivalence class but all of them share the same non-directed edges. 
Theorem 2. For any Markov equivalence class of CGs (under any interpretation), there exists a unique minimal (wrt inclusion) set of non-directed edges that is shared by all members of the class.

The proofs of the Theorem 1 and 2 for the MVR interpretation can be found in the article by Sonntag and Peña [9]. These proofs can easily be adapted for the LWF and AMP interpretations.

\section{Translations between Interpretations}

In this section the main result of this paper is presented, namely what the conditions are for a CG of one interpretation to be possible to translate into a CG of another interpretation. With translate we mean that the induced independence model of a CG of one interpretation can be represented perfectly by a CG of another interpretation. A summary of these results is presented in Table 1.

\begin{tabular}{|c||c|c|c|}
\hline & LWF & AMP & MVR \\
\hline \hline LWF & - & Unidentified & $\begin{array}{c}\left(G_{c l(K)}\right)^{m} \text { is chordal for } \\
\text { all } K \in c c(G) .\end{array}$ \\
\hline AMP & $\begin{array}{c}G \text { contains no } k \text {-biflag } \\
\text { where } k \geq 2[2]\end{array}$ & - & $\begin{array}{c}G^{\prime} \text { does not contain any } \\
\text { induced subgraph of the } \\
\text { form } X-Y-Z\end{array}$ \\
\hline MVR & $\begin{array}{c}G^{\prime} \text { contains no } \\
\text { bidirected edge }\end{array}$ & $\begin{array}{c}G^{\prime} \text { contains no } \\
\text { bidirected flag }\end{array}$ & - \\
\hline
\end{tabular}

Table 1: Given a CG $G$ of the interpretation denoted in the row, and a maximally oriented CG $G^{\prime}$ in the Markov equivalence class of $G$, there exists a CG $H$ of the interpretation denoted in the column st $G$ and $H$ are Markov equivalent iff the condition in the intersecting cell is fulfilled.

From the table two things can be noted. First that the conditions given in the table may include a maximally oriented CG $G^{\prime}$ in the same equivalence class as $G$. This is done for several reasons. First, such a graph is easy and computationally simple to find. Secondly, this allows the proofs to be based on the idea that no feasible split is possible for the interpretation in mind. Third and last, the search space of CGs is smaller and more assumptions can be made on the CG. This in turn allows for more efficient algorithms when calculating if the condition holds for some CG. The second note that can be made is that there still does not exist any necessary and sufficient condition for when a perfect translation of a LWF CG $G$ into an AMP CG $H$ is possible. Andersson et al. gave a necessary condition but also showed that this condition was not sufficient [2]. We have managed to prove the necessity of more elaborate conditions but still been unable to prove sufficiency for these. Hence this condition is left for future work. 
The rest of this section contains the theorems stating the conditions shown in Table 1 together with their proofs.

\subsection{Translation of MVR CGs to AMP CGs}

Theorem 3. Given a MVR $C G G$, and a maximally oriented $M V R C G G^{\prime}$ in the Markov equivalence class of $G$, there exists an $A M P C G H$ st $I_{M V R}(G)=$ $I_{A M P}(H)$ iff $G^{\prime}$ contains no bidirected flag.

Proof. Sufficiency follows from from Lemmas 4 and 5 and necessity follows from Lemma 2.

Lemma 2. A MVR $C G G$ and an $A M P C G H$ with the same structure, except that every bidirected edge in $G$ is replaced by a undirected edge in $H$ and where $G$ contains no bidirected flag, represent the same independence model.

Proof. Assume to contrary that there exists two CGs, $G$ under the MVR interpretation and $H$ under the AMP interpretation, st $G$ does not contain any bidirected flag, i.e induced subgraph of the form $X \leftrightarrow Y \leftrightarrow Z, G$ and $H$ contain the same directed edges, and for every bidirected edge in $G H$ has an undirected edge instead (and only contains those undirected edges) but $I_{M V R}(G) \neq I_{A M P}(H)$. Clearly we must have $V_{G}=V_{H}$ and that $\operatorname{adj}_{G}(X)=\operatorname{adj}_{H}(X), p a_{G}(X)=p a_{H}(X)$ and $c o_{G}(X)=c o_{H}(X)$ holds for all $X \in V_{G}$. Given the definition of strict descendants $\operatorname{san}_{G}(X)=\operatorname{san}_{H}(X)$ must also hold. Moreover note that $H$ cannot contain any induced subgraph of the form $X-Y-Z$. Finally note that both $G$ and $H$ contains the same paths between $X$ and $Y$.

For $I(G) \neq I(H)$ to hold there has to exist a path $\pi$ in $G$ (resp. $H$ ) that is d-connecting (resp. S-open) st there exist no path in $H$ (resp. $G$ ) that is S-open (resp. d-connecting). Let $\pi$ be a minimal d-connecting (resp. S-open) path in $G$ (resp. $H$ ). Note that $\pi$ cannot contain any contain any subpath of the form $V_{1} \leftrightarrow V_{2} \leftrightarrow V_{3}$ (resp. $V_{1}-V_{2}-V_{3}$ ) since the edge $V_{1} \leftrightarrow V_{3}$ (resp. $V_{1}-V_{3}$ ) must exist in $G$ (resp. $H$ ) or $G$ contains a bidirected flag or semi-directed cycle. This in turn would mean that $\pi$ is not minimal since the path $\pi \backslash V_{2}$ also must be dconnecting and shorter than $\pi$. For $\pi$ to be both d-connecting and S-open for any set of nodes $Z$ it must contain the same colliders and head-no-tail nodes. A node $W \in \pi$ is a collider if it is part of the following configurations of edges in $\pi(1) \rightarrow W \leftarrow,(2) \leftrightarrow W \leftarrow,(3) \rightarrow W \leftrightarrow$ and $(4) \leftrightarrow W \leftrightarrow$. Clearly the fourth case cannot occur. Case 1-3 would be translated into (1) $\rightarrow W \leftarrow,(2)-W \leftarrow,(3) \rightarrow W-$ in $H$ which are all (and the only) head-no-tail configurations. Hence $\pi$ must be d-connecting in $G$ iff $\pi$ is S-open in $H$ which contradicts the assumption.

Lemma 3. If a maximally oriented $C G G$ of the MVR interpretation contains a bidirected flag $X \leftrightarrow Y \leftrightarrow Z$ then $G$ also contains an induced subgraph of the form shown in (1) Figure $1 a$ or (2) $1 b$ or (3) $P \circ Q \leftrightarrow Y \leftrightarrow Z$ or (4) $P \circ Q \leftrightarrow W \leftrightarrow Z$ st $b d_{G}(Q) \subseteq b d_{G}(Y) \cup Y$ and $Y \in \operatorname{sp}_{G}(Q)$ hold. 
Proof. Assume the contrary, that no such induced subgraph exists in $G$ even though $G$ contains a bidirected flag and $G$ is maximally orientated. Let $C$ be the component of which $X, Y$ and $Z$ belongs. Let $A$ be the set of nodes $A_{k}$ st $A_{k} \in s p_{G}(Y)$ but $A_{k} \notin s p_{G}(Z)$. We know that $X$ fulfills these criteria and hence $|A| \geq 1$.

First note that if there exists a node $A_{k} \in A$ st $b d_{G}\left(A_{k}\right) \nsubseteq b d_{G}(Y) \cup Y$ then there exists an induced subgraph $P \circ \rightarrow A_{k} \leftrightarrow Y \leftrightarrow Z \cdots P$ in $G$ for some node $P \in b d_{G}\left(A_{k}\right) \backslash b d_{G}(Y) \backslash Y$. Hence we have a contradiction since $G$ either contains an induced subgraph of the form shown in Figure $1 \mathrm{~b}\left(P \in b d_{G}(Z)\right)$ or of the form $P \circ \rightarrow Q \leftrightarrow Y \leftrightarrow Z\left(P \notin b d_{G}(Z)\right)$. Therefore we must have that $b d_{G}\left(A_{k}\right) \subseteq Y \cup b d_{G}(Y)$ holds for all $A_{k} \in A$, i.e. that $b d_{G}(A) \subseteq Y \cup b d_{G}(Y)$ holds.

Secondly note that we can let $B$ be a subset of $A$ st $B$ consists of the nodes in one connected subgraph in the subgraph of $G$ induced by $A$ (any connected subgraph will do). Let $D$ be the set of nodes st $D=s p_{G}(Y) \cap s p_{G}(Z) \cap s p_{G}(B)$. With these sets we know that the spouses of $Y$ can be either adjacent of $Z$ or not, hence $s p_{G}(Y)=D \cup A$ must hold. This in turn gives that $s p_{G}(A)=D \cup Y$ and $b d_{G}(A) \subseteq D \cup Y \cup p a_{G}(Y)$ since $\forall A_{k} \in A b d_{G}\left(A_{k}\right) \subseteq Y \cup b d_{G}(Y)$ holds. Moreover $\operatorname{sp}_{G}(B)=D \cup Y$ and $b d_{G}(B) \subseteq D \cup Y \cup p a_{G}(Y)$ must also hold. Hence, if $D$ is empty then $\operatorname{sp}_{G}(B)=\{Y\}$ and $b d_{G}(B) \subseteq Y \cup p a_{G}(Y)$ must hold. This does however lead to a contradiction because a split then is possible st $U$ consists of $B$ and $L$ consists of $C \backslash U$. Hence there has to exists at least one node in $D$.

Thirdly note that $D \cup Y$ must be complete or the induced subpath $B_{k} \leftrightarrow D Y_{i}$ $\leftrightarrow Z \leftrightarrow D Y_{j} \leftrightarrow B_{1} \leftrightarrow \ldots \leftrightarrow B_{l} \leftrightarrow B_{k}, l \geq 0$, exists in $G$ for some nodes $B_{k}, B_{1}, \ldots, B_{l} \in B$ and $D Y_{i}, D Y_{j} \in D \cup Y$. This means that $G$ contains an induced subgraph of the form shown in either Figure 1a $(l>0)$ or $1 \mathrm{~b}(l=0)$.

Fourth and finally note that there must exist a node $P$ st $P \in b d_{G}(B) \cup B$ but $P \notin b d_{G}\left(D_{j}\right)$ for some $D_{j} \in D \cup Y$ or a split is feasible where $U$ consists of $B$ and $L$ consists of $C \backslash U$. Note that $D_{j} \neq Y$ must hold since $b d_{G}(B) \cup B \subseteq b d_{G}(Y) \cup Y$. This means that there must exist 2 nodes $B_{i}, D_{j}$ st $P \in b d_{G}\left(B_{i}\right), P \notin b d_{G}\left(D_{j}\right), B_{i} \in B$, $B_{i} \in \operatorname{sp}\left(D_{j}\right)$ and $D_{j} \in D$ st the induced subgraph $P \leftrightarrow B_{i} \leftrightarrow D_{j} \leftrightarrow Z \cdots P$ exist in $G$. This is a contradiction either because $G$ contains an induced subgraph of the form shown in Figure 1b $\left(P \in b d_{G}(Z)\right)$ or $P \circ B_{i} \leftrightarrow D_{j} \leftrightarrow Z\left(P \notin b d_{G}(Z)\right)$ where $b d_{G}\left(B_{i}\right) \subseteq b d_{G}(Y) \cup Y$ and $Y \in s p_{G}\left(B_{i}\right)$ holds.

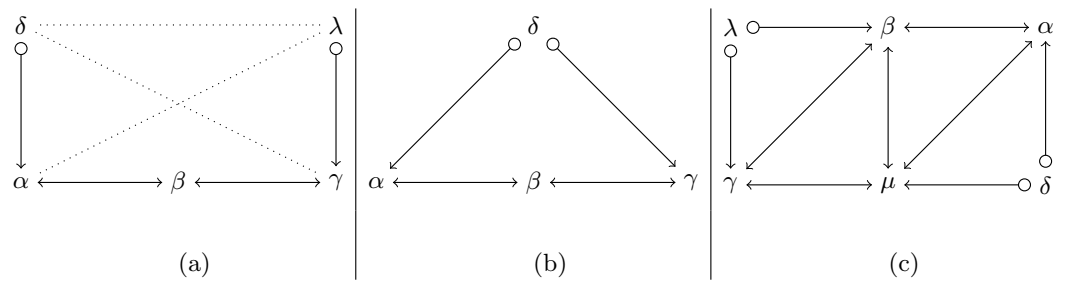

Fig. 1: MVR subgraph forms 
Lemma 4. If a maximally oriented $C G G$ of the MVR interpretation contains a bidirected flag then $G$ at least one of the induced subgraphs shown in Figure 1 exists in $G$.

Proof. Assume the contrary, that no such induced subgraph exists in $G$ even though $G$ contains a bidirected flag and $G$ is maximally orientated. Since $G$ contains a bidirected flag we do with Lemma 3 get that $G$ must contain an induced subgraph $X \leftrightarrow Y \leftrightarrow Z \leftrightarrow W$ or a contradiction directly follows. If we now apply Lemma 3 to $X \leftrightarrow Y \leftrightarrow Z$ we get that, since for $G$ to contain any induced subgraph of the form shown in Figure 1a or $1 \mathrm{~b}$ is a contradiction, there exist a set of nodes (that can be renamed to) $c_{1}, c_{2}, c_{3}$ st the induced subgraph $c_{1} \circ \rightarrow c_{2} \leftrightarrow c_{3} \leftrightarrow Z$ exists in $G$ and $c_{3}=Y$ holds or $b d_{G}\left(c_{2}\right) \subseteq b d_{G}(Y) \cup Y$ and $Y \in s p_{G}\left(c_{2}\right)$ hold. If $c_{3}=Y, G$ must contain the subgraph $c_{1} \circ \rightarrow c_{2} \leftrightarrow Y \leftrightarrow Z \leftrightarrow W$ where $c_{1} \notin a d j_{G}(Y)$ and $W \notin \operatorname{adj}_{G}(Y)$ must hold and $c_{1}=W$ might hold. Clearly this subgraph takes the form of either Figure 1a $\left(c_{1} \neq W\right)$ or $1 \mathrm{~b}\left(c_{1}=W\right)$ which is a contradiction. Hence $c_{3} \neq Y, b d_{G}\left(c_{2}\right) \subseteq b d_{G}(Y) \cup Y$ and $Y \in s p_{G}\left(c_{2}\right)$ must hold.

Since $W \notin \operatorname{adj}_{G}(Y)$ holds and $b d_{G}\left(c_{2}\right) \subseteq b d_{G}(Y) \cup Y$ it is clear that $c_{1}, c_{3} \epsilon$ $b d_{G}(Y)$ must hold. Hence $W \neq c_{2}$ holds since $W \notin \operatorname{adj}_{G}(Y) \cup Y$. This in turn means that $W \notin b d_{G}\left(c_{2}\right)$ holds since $b d_{G}\left(c_{2}\right) \subseteq b d_{G}(Y) \cup Y$ and $W \notin b d_{G}(Y) \cup Y$. Finally we can see that $W \in b d_{G}\left(c_{3}\right)$ holds or the induced subgraph $c_{1} \circ \rightarrow c_{2} \leftrightarrow c_{3} \leftrightarrow Z$ $\leftarrow W$ takes the form shown in Figure $1 \mathrm{a}\left(c_{1} \neq W\right)$ or $1 \mathrm{~b}\left(c_{1}=W\right)$. However, if $W \in b d_{G}\left(c_{3}\right)$ holds $G$ contains an induced subgraph of the form shown in Figure 1c (where $\delta=W, \lambda=c_{1}, \mu=c_{3}, \gamma=c_{2}, \beta=Y$ and $\alpha=Z$ ) and we have a a contradiction.

Lemma 5. The independence model of a $C G G$ of the $M V R$ interpretation which contains an induced subgraph of one of the forms shown in Figure 1 cannot be perfectly represented as a $C G H$ of the AMP interpretation.

Proof. Assume the contrary, that there exists a CG $H$ under the AMP interpretation that can represent these independence models.

First assume that the independence model of the graph shown in Figure 1a can be represented in a CG $H$ of the AMP interpretation. It is clear that $H$ must have the same skeleton, or clearly some separations or non-separations that hold in $G$ would not hold in $H$. The following independence statements holds in $G$ : $\delta \perp_{G} \beta\left|p a_{G}(\beta), \alpha \perp_{G} \gamma\right| p a_{G}(\alpha)$ and $\beta \perp_{G} \lambda\left|p a_{G}(\beta) . \delta \perp_{G} \beta\right| p a_{G}(\beta)$ gives us that a triplex $(\{\delta, \beta\}, \alpha)$ must exist in $H$, since $\alpha \notin p a_{G}(\beta)$ i.e. that (1) $\delta \rightarrow \alpha-\beta$, (2) $\delta-\alpha \leftarrow \beta$ or (3) $\delta \rightarrow \alpha \leftarrow \beta$ exists in $H . \alpha \perp_{G} \gamma \mid p a_{G}(\alpha)$ does however also state that a triplex $(\{\alpha, \gamma\}, \beta)$ must exist in $H$, since $\beta \notin p a_{G}(\alpha)$. For this to happen the edge between $\alpha$ and $\beta$ cannot be orientated towards $\alpha$ hence the subgraph $\delta \rightarrow \alpha-\beta \leftarrow \gamma$ must exist in $H$. The orientation of the edge between $\beta$ and $\gamma$ does however contradict the third independence statement $\beta \perp_{G} \lambda \mid p a_{G}(\beta)$ which implies that the triplex $(\{\beta, \lambda\}, \gamma)$ must exist in $H$, since $\gamma \notin p a_{G}(\beta)$. Hence we have a contradiction if $G$ contains the induced subgraph shown in Figure 1a.

Secondly assume that the independence model of the graph shown in Figure $1 \mathrm{~b}$ can be represented in a CG $H$ of the AMP interpretation. It is clear that $H$ must have the same skeleton, or clearly some separations or non-separations 
that hold in $G$ would not hold in $H$. The following independence statements must then hold in $G: \delta \perp_{G} \beta \mid p a_{G}(\beta)$ and $\alpha \perp_{G} \gamma\left|p a_{G}(\alpha) . \delta \perp_{G} \beta\right| p a_{G}(\beta)$ gives us that two triplexes must exist in $H$, first $(\{\delta, \beta\}, \alpha)$ and secondly $(\{\delta, \beta\}, \gamma)$, since $\alpha, \gamma \notin p a_{G}(\beta) .(\{\delta, \beta\}, \alpha)$ gives that one of the following configurations must occure in $H$ : (1) $\delta-\alpha \leftarrow \beta$, (2) $\delta \rightarrow \alpha-\beta$ or (3) $\delta \rightarrow \alpha \leftarrow \beta$. However, the independence statement $\alpha \perp_{G} \gamma \mid p a_{G}(\alpha)$ implies that the triplex $(\{\alpha, \gamma\}, \beta)$ must exist in $H$ since $\beta \notin p a_{G}(\alpha)$. If the triplex $(\{\alpha, \gamma\}, \beta)$ should hold in $H$ the edge between $\alpha$ and $\beta$ cannot be orientated towards $\alpha$ hence the subgraph $\delta \rightarrow \alpha-\beta \leftarrow \gamma$ must exist in $H$. The orientation of the edge between $\beta$ and $\gamma$ does however contradict the triplex $(\{\delta, \beta\}, \gamma)$ and hence we have a contradiction for the $G$ shown in Figure $1 \mathrm{~b}$.

Third and last assume that the independence model of the graph shown in Figure 1c can be represented in a $\mathrm{CG} H$ of the AMP interpretation. From the Figure we can read the following independence statements: $\lambda_{\perp_{G}} \mu \mid p a_{G}(\mu)$, $\alpha \perp_{G} \gamma\left|p a_{G}(\alpha), \beta \perp_{G} \delta\right| p a_{G}(\beta)$. It is clear that $H$ must have the same skeleton, or clearly some separations or non-separations that hold in $G$ would not hold in $H . \lambda \perp_{G} \mu \mid p a_{G}(\mu)$ and $\alpha \perp_{G} \gamma \mid p a_{G}(\alpha)$ gives that the triplexes $(\{\lambda, \mu\}, \beta)$ and $(\{\alpha, \gamma\}, \mu)$ must exists in $H$ since $\beta \notin p a_{G}(\mu)$ and $\mu \notin p a_{G}(\alpha)$. As seen above this gives that $\lambda \rightarrow \gamma-\mu \leftarrow \alpha$ must exist in $H$. Similarly $\beta \perp_{G} \delta \mid p a_{G}(\beta)$ and $\lambda_{\perp_{G}} \mu \mid p a_{G}(\mu)$ gives that $\lambda \rightarrow \beta-\mu \leftarrow \delta$ must exist in $H$. Finally $\alpha \perp_{G} \gamma \mid p a_{G}(\alpha)$ and $\beta \perp_{G} \delta \mid p a_{G}(\beta)$ gives that the triplexes $(\{\alpha, \gamma\}, \beta)$ and $(\{\beta, \delta\}, \alpha)$ must hold in $H$, since $\beta \notin p a_{G}(\alpha)$ and $\alpha \notin p a_{G}(\beta)$, which in turn gives that $\gamma \rightarrow \beta-\alpha \leftarrow \delta$ must exist in $H$. This does however contradict that $H$ is a $\mathrm{CG}$ since the semi-directed cycle $\gamma \rightarrow \beta-\mu-\gamma$ exists in $H$. Hence we have a contradiction.

\subsection{Translation of AMP CGs to MVR CGs}

Theorem 4. Given an AMP CG G, and a maximally oriented $A M P C G G^{\prime}$ in the Markov equivalence class of $G$, there exists a $C G H$ st $I_{A M P}(G)=I_{M V R}(H)$ iff $G^{\prime}$ does not contain any induced subgraph of the form $X-Y-Z$.

Proof. Sufficiency follows from Lemma 2 while necessity follows from 6.

Lemma 6. If a maximally orientated $C G G$ of the AMP interpretation contains an induced subgraph of the form $X-Y-Z$ then $G$ there exists no $C G H$ of the $M V R$ interpretation st $I_{A M P}(G)=I_{M V R}(H)$.

Proof. Assume to the contrary that the lemma does not hold. Clearly $G$ and $H$ must have the same skeleton or some separations in $H$ do not hold in $G$ or vice versa. Let $H$ have a component ordering ord for its components $c_{1}, \ldots c_{k}$ st $\operatorname{ord}\left(c_{i}\right)<\operatorname{ord}\left(c_{j}\right)$ if $c_{i}$ is a parent of $c_{j}$. Let $C$ be the component of $X$ in $G$. From the assumption we know that $X \perp_{G} Z \mid n b_{G}(X) \cup p a_{G}\left(X \cup n b_{G}(X)\right)$ holds, where $Y \in n b_{G}(X)$, and hence that $H$ must contain one of the following induced subgraphs: $X \leftrightarrow Y \rightarrow Z, X \leftarrow Y \leftarrow Z$ or $X \leftarrow Y \rightarrow Z$. For any other configuration of edges $X \perp_{H} Z \mid n b_{G}(X) \cup p a_{G}\left(X \cup n b_{G}(X)\right)$ does not hold. Moreover we can generalize the configurations to $X \circ Y \rightarrow Z$ and $X \leftarrow Y \rightarrow Z$ simply by choosing the nodes to represent $X$ and $Z$ accordingly. Both these structures are included in $X \circ{ }_{\text {a }} \circ Z$ 
and we will now show that this structure leads to a contradiction if a split is not feasible in $G$.

The proof is iterative and when a restart is noted this is where the proof restarts. For each restart it will be shown that there must exist a triplet of nodes $X, Y, Z$ st an induced subgraph of the form $X-Y-Z$ exists in $G$ and $X \circ \backsim Y \rightarrow Z$ in $H$. Apart from this we also know that $I_{A M P}(G)=I_{M V R}(H)$ holds and that no split is feasible in $G$. Let the set $U$ consist of $Y$ and every node connected by a path to $Y$ in the subgraph of $G$ induced by $C \backslash Z$ and the set $L$ consist of $C \backslash U$. This separation of sets gives that $n b_{G}(U)=Z$. For a split not to be feasible with these sets one of the conditions in Definition 2 must fail:

Case 1, condition 1 or 2 fails. This means that there exist two nodes $W \in C$ and $P \in C$ st the induced subgraph $P-Z-W$ exists in $G$. Note that one of the nodes might be $Y$. This means that $P \perp_{G} W \mid n b_{G}(W) \cup p a_{G}\left(W \cup n b_{G}(W)\right)$ holds, where $Z \in n b_{G}(W)$ and hence that $P \circ Z \rightarrow W, P \leftarrow Z \leftarrow W$ or $P \leftarrow Z \rightarrow W$ must exist in $H$ as described above. Without losing generality we can say that either $P \circ Z \rightarrow W$ or $P \leftarrow Z \rightarrow W$ exists in $H$ and that $W \neq Y$ by choosing $P$ and $W$ appropriately. This means that we can restart the proof with the structure $P \circ \backsim \rightarrow W$ in $H$ (and $P-Z-W$ in $G$ ). The number of restarts is bounded since (1) the number of nodes in $V$ is bounded and that $\operatorname{ord}\left(\mathrm{co}_{H}(Z)\right)>\operatorname{ord}\left(\mathrm{co}_{H}(Y)\right)$.

Case 2, condition 1 and 2 hold but condition 3 fails. This means that there exists two nodes $W \in U$ and $P \notin C$ st the induced subgraph $Z-W \leftarrow P$ exists in $G$. First let us cover the case where $W=Y$. This means that $Z \perp_{G} P \mid p a_{G}(Z)$ holds. Since $H$ have the same skeleton as $G$ this means that $H$ must contain an induced subgraph of the form $P \circ \rightarrow Y \leftarrow Z$ since $Y \notin p a_{G}(Z)$. At the same time we know that $H$ contains the edge $Y \rightarrow Z$ which causes a contradiction and hence $Y \neq W$ must hold. Therefore, $P \notin p a_{G}(Y)$ holds which generalized means that that $p a_{G}(Y) \subseteq p a_{G}(Z)$ must hold. For $Z \perp_{G} P \mid p a_{G}(Z)$ to hold in $H$ there must exist an unshielded collider between $Z$ and $P$ over $W$ and hence that the induced subgraph $Z \circ W \hookleftarrow P$ exists in $H$. Similarly we have that $Y \perp_{G} P \mid p a_{G}(Y)$ gives that $H$ contains an induced subgraph of the form $Y \circ W \leftrightarrow P$. Note that $Y \in \operatorname{adj}_{G}(W)$ must hold since condition 2 holds. Moreover for $H$ not to contain a semi directed cycle over $Y \rightarrow Z \circ W \hookleftarrow Y$ we can see that $Y \rightarrow W \hookleftarrow P$ must exist in $H$. Finally note that $X \neq W$ must hold since $X \notin \operatorname{adj}_{G}(Z)$ holds.

Now assume $X \in n b_{G}(W)$. For $X \perp_{H} Z \mid n b_{G}(X) \cup p a_{G}\left(X \cup n b_{G}(X)\right)$ to hold, together with $W \in n b_{G}(X)$ and $Z \circ \rightarrow W$, it is easy to see that the induced subgraph $Z \circ W \rightarrow X$ must be in $H$. We can now see that $P \in p a_{G}(X)$ must hold or the induced subgraph $X \leftarrow W \leftarrow P$ in $H$ contradicts that $X \perp_{G} P \mid p a_{G}(X)$ holds in $H$. Moreover, for $X \circ \multimap Y \rightarrow W \rightarrow X$ not to form a semi-directed cycle in $H$ the edge between $X$ and $Y$ must be orientated to $X \leftarrow Y$. We can therefore restart the proof by replacing $X$ with $Z$, i.e. with the induced subgraph $X \leftarrow Y \circ \multimap Z$ in $H$ (and $X-Y-Z$ ) in $G$. Since we know that $Z \circ \rightarrow W \rightarrow X$ exists in $H$ we know that $\operatorname{ord}\left(\operatorname{co}_{H}(X)\right)>\operatorname{ord}\left(\operatorname{co}_{H}(Z)\right)$. Hence we cannot get back to this subcase again (or we would have that $\operatorname{ord}\left(\mathrm{co}_{H}(X)\right)<\operatorname{ord}\left(\mathrm{co}_{H}(Z)\right)$ which is a contradiction). This, together with that $Y$ is kept the same and that $|V|$ is finite gives that the number of restarts is bounded. Hence $X \notin n b_{G}(W)$ must hold. 
Now assume that $p a_{G}(Z) \subseteq p a_{G}(W)$. We can now restart the proof with $X \circ \multimap Y \rightarrow W$. The number of iterations is then bounded since $|V|$ is finite and case 2 cannot occur with $Z$ as $W$ again, or $p a_{G}(Z) \nsubseteq p a_{G}(W)$ would have to hold which is a contradiction. Hence $p a_{G}(Z) \nsubseteq p a_{G}(W)$ must hold. Let $Q$ be the parent of $Z$ not shared by $W$. Since $W \perp_{G} Q \mid p a_{G}(W)$ holds, and we know that $H$ contains the induced subgraph $Q \curvearrowleft Q_{\circ} \rightarrow W \hookleftarrow P$, we can draw the conclusion that $H$ must contain the induced subgraph $Q \circ Z \leftrightarrow W \leftrightarrow P$ since $Z \notin p a_{G}(W)$. Note that if there exist two different nodes $W_{1}$ and $W_{2}$ such that both have the properties described for $W$ in case $2 W_{1}$ and $W_{2}$ must be adjacent. If this were not the case we would have that both $W_{1} \perp_{G} W_{2} \mid n b_{G}\left(W_{1}\right) \cup p a_{G}\left(W_{1} \cup n b_{G}\left(W_{1}\right)\right)$ and $W_{1} t_{H} W_{2} \mid n b_{G}\left(W_{1}\right) \cup p a_{G}\left(W_{1} \cup n b_{G}\left(W_{1}\right)\right)$ would hold, since $Z \in n b_{G}\left(W_{1}\right)$. Also note that since $W_{1} \leftrightarrow Z$ and $W_{2} \leftrightarrow Z$ exists in $H$ and the edge between $W_{1}$ and $W_{2}$ must be bidirected or $H$ contains a semi-directed cycle. Let $D$ be a set of nodes containg $Z$ as well as all nodes that have the properties described for $W$. From the description above we can see that $D$ must be complete and that the subgraph induced by $D$ in $H$ must only contain bidirected edges. We will now show that a split must be feasible in $G$ with $D$ as $L$ and $C \backslash D$ as $U$. For a split not to be feasible one of the constraints in Definition 2 must fail.

Assume condition (1) or (2) fails. Then there exists three nodes $R \in C$, $T \in C$ and $D_{j} \in D$ st the induced subgraph $T-D_{j}-R$ exists in $G$. Since $T \perp$ ${ }_{G} R \mid n b_{G}(R) \cup p a_{G}\left(R \cup n b_{G}(R)\right)$ holds we must, without losing generalization, have that $H$ contains the induced subgraph $T \circ \multimap D_{j} \rightarrow R$, since $D_{j} \in n b_{G}(R)$. If this is the case we can however restart the proof with this induced subgraph and know that the number of iterations is bounded since $|V|$ is finite and $\operatorname{ord}\left(\operatorname{co}_{H}\left(D_{j}\right)\right)>$ $\operatorname{ord}\left(\mathrm{co}_{H}(Y)\right)$.

Assume condition (1) and (2) holds but (3) fails. Then there exists two nodes $R \in U$ and $T \notin C$ st the induced subgraph $D_{i}-R \leftarrow T$ exists in $G$ for some $D_{i} \in D$. First note that $R$ must be adjacent of all nodes in $D$ or condition 1 would have failed in this split. Secondly note that $R-Y$ must exist in $G$ or condition 2 would fail if we restart the proof with $X \circ \circ Y \rightarrow D_{i}$ and a contradiction follows from there. Thirdly note that $R \notin \operatorname{adj}_{G}(X)$ must hold or the proof could be restarted with $X \multimap \circ Y \rightarrow D_{i}$, for which condition 3 would fail with $R$ as $W$ and a contradiction would follow as shown above. Finally note that $p a_{G}(R) \subseteq p a_{G}(Z)$ must hold or $R$ would be in $D$. This means that $p a_{G}(W) \notin p a_{G}(R)$, and hence that $P \notin p a_{G}(R)$, holds. Moreover we know that the edge $D_{i} \leftrightarrow W$ exists in $G$. For $D_{i} \perp_{G} T \mid p a_{G}\left(D_{i}\right)$ to hold in $H$ it is clear that $H$ must contain the induced subgraph $D_{i} \circ R \leftarrow T$ since $R \notin p a_{G}\left(D_{i}\right)$. Similarly we have that for $R \perp_{H} P \mid p a_{G}(R)$ to hold $H$ must contain an induced subgraph of the form $R \leftrightarrow W \leftrightarrow P$ since $W \notin p a_{G}(R)$. This means that for $R \leftrightarrow W \leftrightarrow D_{i} \rightarrow R$ not to form a semi-directed cycle in $H$ the edge $R \leftrightarrow D_{i}$ must exist in $H$. Moreover, since $\forall D_{m} \in D \backslash D_{i} R \in \operatorname{adj}_{G}\left(D_{m}\right), R \leftrightarrow D_{i}$ and $D_{i} \leftrightarrow D_{m}$ hold, clearly $R \leftrightarrow D_{m}$ must also hold or $G$ contains a semi-directed cycle. Hence the subgraph of $H$ induced by $D \cup R$ is complete and contains only bidirected edges. This in turn means that for $Y \rightarrow D_{i} \leftrightarrow R \circ \multimap Y$ to not form a semi-directed cycle $Y \rightarrow R$ must exist in $H$. 
Hence we can move $R$ into $D$ and redo do the last split again. The number of restarts are bounded since $|V|$ is finite.

Hence each condition in Definition 2 must hold and we have a contradiction.

\subsection{Translation of MVR CGs to LWF CGs}

Theorem 5. Given a MVR $C G G$, and a maximally oriented $M V R C G G^{\prime}$ that is in the same Markov equivalence class as $G$, there exist a LWF CG H st $I_{M V R}(G)=I_{L W F}(H)$ iff $G^{\prime}$ contains no bidirected edge, i.e. can be represented as a $B N$.

Proof. From Lemma 7 it follows that a maximally oriented CG $G^{\prime}$ of the MVR interpretation with a bidirected edge must have a subgraph of the form shown in Figure 2. If it does not contain any bidirected edge in the maximally oriented model it trivially follows that it is a $\mathrm{BN}$ (and hence it can be represented as a CG of the LWF interpretation). From Lemma 8 it then follows that no CG $G$ of the MVR interpretation which contains a subgraph of the form shown in Figure 2 can be represented as a CG of the LWF interpretation.

Lemma 7. If a bidirected edge exists in a maximally oriented $C G G$ of the MVR interpretation then $G$ must contain an induced subgraph of the form shown in Figure 2.

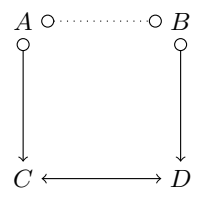

Fig. 2: Included subgraph in Lemma 7 and 8.

Proof. Assume to the contrary that a CG $G$ of the MVR interpretation exists where (1) no induced subgraph of the form shown in Figure 2 exists, (2) no split is feasible and (3) at least one bidirected edge exists. From this assumption we can see that there has to exist at least two nodes $X$ and $Y$ st $X \leftrightarrow Y$ exists in $G$. Let $C$ be the connectivity component to which $X$ and $Y$ belongs. Separate the nodes of $C$ into two sets $U$ and $L$ st $X$ and every node connected by a path to $X$ in the subgraph of $G$ induced by $C \backslash Y$ belongs to $L$ and $C \backslash L$ belongs to $U$. This separation of nodes allows us to know that $s p_{G}(L)$ only contains $Y$. For a split not to be feasible at least one condition in Definition 3 has to fail.

Case 1. Assume constraint 1 fails. This means a node $Z \in L$ exist st $Z \leftrightarrow Y \leftrightarrow X$ occurs in $G$ where $Z \notin \operatorname{adj}_{G}(X)$ must hold, or $Z$ would be in $U$. Now remove $Y$ from $U$ and add it to $L$ as well as all nodes not connected by a path with $Z$ in the subgraph of $G$ induced by $U \backslash Y$ and attempt another split. This 
separation of nodes allows us, since we previously had $n b_{G}(L)=Y$ and $Y$ now have changed sets, to say that $s p_{G}(U)=Y$ must hold and hence that constraint 3 cannot fail. However, if constraint 1 or 2 fails we know there exists a node $W$ st $W \circ Z \leftrightarrow Y \leftrightarrow X$ is a subgraph of $G$ but where $W \notin a d j_{G}(Y)$, and $Z \notin a d j_{G}(X)$ and $W \notin \operatorname{adj}_{G}(X)$ by definition of the initial split, which implies a contradictory induced subgraph. Hence constraint 1 cannot fail in the initial split.

Case 2. If constraint 2 or 3 fails in the initial split we know there exists two nodes $V_{1}$ and $P_{1}$ st $P_{1} \circ Y \leftrightarrow V_{1}$ exists in $G$ but where $V_{1} \notin a d j_{G}\left(P_{1}\right)$ (note that $V_{1}$ might be $X$ ). Now let $L$ consist of every node connected by a path to $Y$ in the subgraph of $G$ induced by $C \backslash V_{1}$ and the nodes $C \backslash L$ belong to $U$. This separation of nodes allows us to know that $s p_{G}(L)$ only contains $V_{1}$. If constraint 1 fails when performing a split with these sets it is clear from case 1 that a contradiction occurs. If constraint 2 or 3 fails we know there exists two new nodes $V_{2}$ and $P_{2}$ st $P_{2} \circ V_{1} \leftrightarrow V_{2}$ exists in $G$ but where $P_{2} \notin a d j_{G}\left(V_{2}\right)$. Note that $V_{2}$ or $P_{2}$ cannot be $P_{1}$ since $P_{1} \notin a d j_{G}\left(P_{1}\right)$. We now get that $V_{2}$ cannot be $Y$ or an induced subgraph like that in Figure 2 occurs. $V_{2} \in \operatorname{adj}(Y)$ and $P_{2} \in \operatorname{adj}(Y)$ must also hold or the induced subgraph $V_{2}$ (resp. $\left.P_{2}\right) \leftrightarrow V_{1} \leftrightarrow Y \leftarrow P_{1}$ occurs. By replacing $V_{1}$ with $V_{2}$, setting the proper $U$ and $L$ as described above it we can now repeat this procedure iteratively. Moreover, for every repetition $i$ we must have that $V_{i}$ and $P_{i}$ must be adjacent of every $V_{j}(j<i)$ as well as $Y$ or a contradiction occurs. This means that any nodes $V_{i}$ and $P_{i}$ already used in a previous repetition cannot be used in a later one, or both $P_{i} \in \operatorname{adj}\left(V_{i}\right)$ and $P_{i} \notin \operatorname{adj}\left(V_{i}\right)$ would have to hold. This in turn means that the number of repetitions is bounded since $|C|$ is finite and hence we have a contradiction that condition 2 or 3 can fail.

This means that all three conditions in Definition 3 must hold and hence a split must be feasible if the induced subgraph shown in Figure 2 does not occur.

Lemma 8. If a $C G G$ of the MVR interpretation contains an induced subgraph of the form shown in Figure 2 then $G$ cannot be translated into a $C G H$ of the LWF interpretation.

Proof. Assume to the contrary that there exists a CG $H$, of the LWF interpretation, with the same independence model as $G$ while $G$ contains an induced subgraph of the form shown in Figure 2. Clearly $H$ and $G$ must contain the same nodes and adjacencies or some separations or non-separations must exist in $G$ but not in $H$.

From Figure 2 we can read that $A \perp_{G} D \mid p a_{G}(D)$ and $C \perp_{G} B \mid p a_{G}(C)$ hold. For $A \perp_{G} D \mid p a_{G}(D)$ to hold in $H C$ must be a collider between $A$ and $D$ and hence $H$ must contain the induced subgraph $A \rightarrow C \leftarrow D$. Similarly $C \perp_{G} B \mid p a_{G}(C)$ gives that $H$ must contain the induced subgraph $C \rightarrow D \leftarrow B$ and hence we have a contradiction.

\subsection{Translation of LWF CGs to MVR CGs}

Theorem 6. Given a $L W F C G G$ there exists a $C G H$ st $I_{L W F}(G)=I_{M V R}(H)$ iff $\left(G_{c l(K)}\right)^{m}$ is chordal for all $K \in c c(G)$. 
Proof. To prove the "if" part, note that if $\left(G_{c l(K)}\right)^{m}$ is chordal for all $K \in c c(G)$, then there is a DAG $D$ st $I_{L W F}(G)=I_{B N}(D)$ [1, Proposition 4.2] and, thus, it suffices to take $H=D$.

To prove the "only if" part, assume to the contrary that $V_{1}-\ldots-V_{n}$ is a chordless undirected cycle in $\left(G_{c l(K)}\right)^{m}$ for some $K \in c c(G)$. Note that $H$ has the same adjacencies as $G$. Therefore, $V_{i-1} \leftarrow V_{i}$ and/or $V_{i} \rightarrow V_{i+1}$ must be in $H$ because, otherwise, $V_{i-1} \perp_{G} V_{i+1} \mid Z \in I_{L W F}(G)$ for some $Z$ st $V_{i} \in Z$ whereas $V_{i-1} \perp_{H} V_{i+1} \mid Z \notin I_{M V R}(H)$, which contradicts that $I_{L W F}(G)=I_{M V R}(H)$. Assume without loss of generality that $V_{i} \rightarrow V_{i+1}$ is in $H$. Then, $V_{i+1} \rightarrow V_{i+2}$ must be in $H$ too, by an argument similar to the previous one. Repeated application of this reasoning implies that $H$ has a semi-directed cycle, which contradicts the definition of CG.

\section{Acknowledgments}

This work is funded by the Center for Industrial Information Technology (CENIIT) and a so-called career contract at Linköping University, by the Swedish Research Council (ref. 2010-4808), and by FEDER funds and the Spanish Government (MICINN) through the project TIN2010-20900-C04-03.

\section{References}

1. Andersson, S.A., Madigan, D., Pearlman, M.D.: A Characterization of Markov Equivalence Classes for Acyclic Digraphs. Annals of Statistics 25:2, 505-541 (1997)

2. Andersson, S.A., Madigan, D., Pearlman, M.D.: An Alternative Markov Property for Chain Graphs. Scandianavian Journal of Statistics 28, 33-85 (2001)

3. Cox, D.R., Wermuth, N.: Linear Dependencies Represented by Chain Graphs. Statistical Science 8, 204-283 (1993)

4. Cox, D.R., Wermuth, N.: Multivariate Dependencies: Models, Analysis and Interpretation. Chapman and Hall (1996)

5. Drton, M.: Discrete Chain Graph Models. Bernoulli 15(3), 736-753 (2009)

6. Frydenberg, M.: The Chain Graph Markov Property. Scandinavian Journal of Statistics 17, 333-353 (1990)

7. Lauritzen, S.L., Wermuth, N.: Graphical Models for Association Between Variables, Some of Which are Qualitative and Some Quantitative. Annals of Statistics 17, 3157 (1989)

8. Roverto, A., Studený, M.: A Graphical Representation of Equivalence Classes of AMP Chain Graphs. Journal of Machine Learning Research 7(6), 1045-1078 (2006)

9. Sonntag, D., Peña, J.M.: Learning Multivariate Regression Chain Graphs under Faithfulness. In: Proceedings of the 6th European Workshop on Probabilistic Graphical Models. pp. 299-306 (2012)

10. Studený, M., Roverto, A., Štěpánová, Š.: Two Operations of Merging and Splitting Components in a Chain Graph. Kybernetika 45, 208-248 (1997)

11. Wermuth, N., Sadeghi, K.: Sequences of Regression and their Indepdendences. TEST 21(2), 215-252 (2012)

12. Wermuth, N., Wiedenbeck, M., Cox, D.: Partial Inversion for Linear Systems and Partial Closure of Independence Graphs. BIT Numerical Mathematics 46, 883-901 (2006) 